\title{
Lyophilization-free proliposomes for sustained release oral delivery of hydrophobic drug (cinnarazine): a comparative study
}

https://doi.org/10.1515/pthp-2021-0002

Received February 16, 2021; accepted May 18, 2021;

published online May 28, 2021

\section{Abstract}

Objectives: Cinnarizine is used for the treatment of vestibular disorders. However, its poor solubility limits its clinical uses due to many challenges. Liposomes were utilised to improve the release profile of many poorly soluble drugs. However, liposomes face many stability challenges during the storage period. This study aims to develop proliposomes designed for the oral delivery of cinnarizine with enhanced stability characteristics.

Methods: Three cinnarizine entrapping Proliposomal formulations were prepared with different ingredients and compared with their liposomal counterparts. Both vesicular approaches were characterised for their particle size, encapsulation efficiency, drug release and stability.

Results: The proliposomes were superior to liposomes in their stability and release profiles. Although no significant changes were noticed between the encapsulation efficiency percentage of the liposomal and proliposomal formulations on the day of preparation, storing the formulations for two weeks ended up with significant leakage of the drug from liposomes $(p<0.05)$ due to stability issues, but not in proliposomes. Moreover, the proliposomes released $100 \%$ of cinnarizine throughout the dissolution experiment in gastric fluid in comparison with the total released drug of $70 \%$ from the liposomes.

Conclusions: Proliposomes provided a successful approach to deliver lipophilic drugs orally to improve their

\footnotetext{
*Corresponding author: Omar S. Abu Abed, Department of Health Sciences, Arab American University, Al-Reehan, Ramallah 91000, Palestine; and Department of Pharmacy and Wellbeing, University of Sunderland, Sunderland, UK, E-mail: omar.abuabed@aaup.edu. https://orcid.org/0000-0002-7118-2441

Srilikha Mulkala, Asma M. Abdin and Amal A. Elkordy, Department of Pharmacy and Wellbeing, University of Sunderland, Sunderland, UK Israa Sharif, Department of Health and Nutrition, Palestine Polytechnic University, Hebron, Palestine. https://orcid.org/00000002-1195-2221
}

pharmacokinetic properties by converting their crystalline nature into more amorphous agents.

Keywords: liposomes; proliposomes; lipid delivery systems; cinnarizine; oral delivery.

\section{Introduction}

Cinnarizine is a first-generation antihistamine. One of the main side effects of cinnarizine is the induced drowsiness which became the main therapeutic indications due to its central effect in managing vestibular disorders, e.g. vertigo, Meniere's disease, and tinnitus [1, 2]. Cinnarizine is a class II drug according to the biopharmaceutical classification system (BCS); it has very good permeation and poor solubility, which may limit its oral uses [3]. Despite the long half-life cinnarizine has, the frequency of administration is three to four times a day as a result of its limited solubility as the GI fluid is not able to dissolve such a high amount of the drug all at once. Cinnarizine is well absorbed in the upper gastrointestinal tract (GIT) due to its high lipophilic properties; however, the same properties limit the solubility and the readiness for absorption as a limiting step [4]. Therefore, enhancing the drug solubility and dissolution profile may be the key to improving the pharmacokinetic properties of the therapeutic agent. Many techniques are utilised to overcome the solubility limitations of cinnarizine, e.g. niosome [5] and complexation and conjugation [6]. Nanotechnology-based drug delivery systems opened new horizons for the oral delivery of many small drugs and macromolecules [7, 8]. Liposomes [9], polymeric nanoparticles [10], niosomes [11], and nanoemulsions [12] are considered the most common examples of nanocarriers that are utilised for oral delivery.

Liposomes are bilayer phospholipid spherical vesicles with aqueous and lipophilic domains. The amphipathic nature of liposomes makes them great potential delivery systems for a wide variety of active pharmaceutical ingredients. Liposomes have multipurpose structure and intrinsic properties that provided them with advanced applications. The popularity of liposomes is owed to their meritorious features; biocompatibility, biodegradability, non-immunogenicity, 
and safety [13]. Many studies revealed the prominent roles that liposomes play in the field of drug delivery systems. Liposomes have great potential in enhancing drug solubility, providing targeted delivery systems, modifying and extending drug release, and improving the safety profile and pharmacokinetic properties of delivered drugs $[14,15]$. However, physical, chemical and biological stability issues burden the successful development of liposomes due to many processes that happen during the storage and delivery of liposomes which may induce phospholipid hydrolysis and oxidation and vesicles aggregation, sedimentation and fusion $[5,16]$. Therefore, the approaches for stabilising phospholipid vesicles are emerging and necessary to enhance the clinical and preclinical applications of liposomes; e.g. Polyethylene glycol functionalisation [17] and using synthetic phospholipids with boosted stabilising properties [18].

Proliposomes are a promising approach to improve physicochemical properties, release profile and pharmacokinetic characteristics, and the stability of liposomes [19, 20]. Proliposomes are dry flowable particles that produce a suspension of liposomes when reconstituted with aqueous solvents [21]. Proliposomes have been advocated to overcome the limitations of the oral delivery of lipophilic drugs by promoting their absorption, owing to their interaction with bile salts in the GIT to form mixed micellular system of vehicles/mesophases and convert the intrinsic nature of some drugs from being crystalline into agents with more amorphous properties [22-25].

In this study, we prepared proliposomal and liposomal formulations containing different lipid: cholesterol ratios with and without cremophor by using the thin lipid film hydration method. All formulations were characterised for their physicochemical properties and release profile. This study aimed to develop and characterise proliposomal formulations designed for the oral delivery of cinnarizine. A critical comparison between the proliposomal formulations and their liposomal counterparts were held.

\section{Materials and methods}

\section{Materials}

Lipid 1,2-Distearoyl-sn-glycerol-3-phosphocholine (DSPC), cholesterol, cinnarizine was purchased from all known company SigmaAldrich. The cosurfactant cremophor ${ }^{\circledR}$ EL (polyethoxylated castor oil) was purchased from BASF Aktiengesellschaft (Ludwigshafen, Germany). The solvents; chloroform, dichloromethane, and hydrochloric acid were from Fischer Chemicals and freshly prepared distilled water was used for the further dilutions of $0.1 \mathrm{~N} \mathrm{HCl}$.

\section{Methods}

Liposomes and proliposomes were formulated with different lipid: cholesterol ratios with or without cremophor by using the thin lipid film hydration method. Liposomes were prepared as described earlier by [14]. Briefly, individual solutions of lipid, cremophor, cholesterol, or cinnarizine in chloroform were prepared and transferred into a round-bottom flask. Then, the organic solvent was evaporated by a rotary evaporator (Rotavapor R205, Buchi Labortechnik AG, Switzerland) at $60^{\circ} \mathrm{C}, 100 \mathrm{rpm}$, and $464 \pm 10 \mathrm{mbar}$ pressure until a thin film was produced. The obtained film was hydrated with $0.1 \mathrm{~N} \mathrm{HCl}$ in a shaking water bath at $60^{\circ} \mathrm{C}$ for $1 \mathrm{~h}$ and left to cool down. Afterwards, the systems were bath sonicated for $10 \mathrm{~min}$ to reduce and uniform the particle size by producing unilamellar vesicles. The free drug was separated from the vesicles by passing the suspension through size exclusion Sephadex ${ }^{\mathrm{TM}}$ G-25 Medium columns (Prepacked PD-10 Desalting, GE); all illusion fractions were collected. The last fractions that contain liposomes were characterised and kept for later stability tests. For the proliposomal formulations, the suspensions were centrifugated and washed in distilled water twice at 15,000 rpm, $4^{\circ} \mathrm{C}$, for $30 \mathrm{~min}$. The supernatants were discarded, and the pellets were collected and kept in Eppendorf inside a bucket of ice and dried under the fume cupboard for $24 \mathrm{~h}$. Afterwards, the obtained powders were sieved through \#120 mesh to improve the flowability of the proliposomal formulations and break down any aggregates to produce uniform particles.

Then, the prepared formulations were characterised for their physicochemical properties, drug release and 14 days storage stability. The proliposomes were resuspended on the day of characterisation by adding $5 \mathrm{ml}$ prewarmed saline and hand-shaking the samples until they are entirely homogeneous. Particle size analysis for all formulations was investigated by using Zeta PALS (Brookhaven Instruments Corporation, USA), particle size in $\mathrm{nm}$ and PDI results are the average of triplicate readings. Size analysis for all formulations was performed on the day of preparation by further diluting the samples and vertexing them for uniformity. Moreover, the encapsulation efficiency of all formulations was investigated on the same day of preparation and after 14 days of storage at chilled conditions $\left(5^{\circ} \mathrm{C} \pm 2\right)$. The rationale of measuring the encapsulation efficiency after storing the formulations was to examine the leakage of the drug from the vesicles in liposomes and proliposomes.

Diluted $1 \mathrm{ml}$ of suspension in $0.1 \mathrm{~N} \mathrm{HCl}$ was diluted with $2 \mathrm{ml}$ of chloroform to dissolve liposome ingredients. Once the systems turned into a clear solution, the absorbance was measured at $\lambda=253 \mathrm{~nm}$ using a single-beam UV/Vis Spectrophotometer (Camspec M501, UK) against 1:2 HCl: chloroform as a blank reference. The release profile of both vesicles was investigated in simulated gastric fluid (SGF) to assess their potential to release the drug in the stomach. The dissolution study was carried out in Eppendorf vials $(1.25 \mathrm{ml})$ for a total time of $4 \mathrm{~h}$. One vial for each time point of every sample was allocated in a shaking water bath at $37 \pm 0.5^{\circ} \mathrm{C}$ with $100 \mathrm{rpm}$. At the end of each time point, the vials were collected, washed twice by centrifugation at $10,000 \mathrm{rpm}$, and the absorbance at $\lambda=253 \mathrm{~nm}\left(\mathrm{~A}_{253}\right)$ was measured. The concentration was calculated by substituting the obtained absorbance in the generated equation by plotting a series of concentrations $(2-20 \mu \mathrm{g} / \mathrm{ml}$ drug in $0.1 \mathrm{~N} \mathrm{HCl})$ vs $\mathrm{A}_{253}$ by utilising a single beam UV/Vis Spectrophotometer, Eq. (1).

$$
y=0.594 x+0.0269 \quad R^{2}=0.9988
$$


All results are presented as the mean of triplicate \pm standard deviation (SD). Paired sample T-tests were performed, and the twotailed significance ( $p$-value) was determined, and $p$-value $\leq 0.05$ was considered statistical significance.

\section{Results and discussion}

DSPC phospholipid was selected in this study due it its proven superiority in physicochemical properties over other phospholipids; namely, DMPC and DPPC. The rigidity of DSPC makes it a great potential for formulating stable liposomes for in vivo administration and for withstanding throughout storage [23]. Cholesterol was used at different amounts for its role in reducing the fluidity of the liposomal shell and increasing their packing density and mechanical properties [26]. Liposomes and proliposomes were prepared with different lipid: cholesterol ratios. Liposomes were kept and stored as suspensions in Phosphate buffer saline as the acidic media may enhance the leakages of cinnarizine to the outer shell due to its good solubility at low $\mathrm{pH}$ values and poor solubility at $\mathrm{pH}$ values above 6 [27]. The Proliposomal formulations were dried and formed dry and fluffy whitish powder and turbid milky suspension upon reconstitution. The particle size analysis revealed that all formulations were in the nanoscale with no significant effect of changing the ingredients, and their amount was reported. However, the particle size of the proliposomes was significantly larger than their counterpart liposomes, Table1. The large particle size of proliposomes is attributed to the formation of multilamellar vesicles upon reconstitution with water as reported by [23]. The multilamellar vesicles have larger intra-layer spaces that can be considered as a lipophilic domain suitable for entrapping poorly soluble drugs.
According to the FDA, PDI is an essential component and critical quality attribute that should usually be considered in evaluating liposomal drug products. The recorded PDI ranges from 0.1-0.3 for all formulations without extreme readings. In liposomal formulations, PDI readings less than 0.3 are acceptable and represent homogenous colloidal systems. In contrast, polymer-based nanocarriers should have PDI values less than 0.2 to be considered acceptable [28]. PDI readings were changed after the period of storage; however, all of them remained within the same range (0.1-0.3).

This feature may increase the amount of the encapsulated cinnarizine in proliposomes upon contact with water and body fluids. The encapsulation efficiency values were similar for all formulation when measured on the same day of formulations. The formulations that do not contain cremophor (L3 and PL3) showed slightly higher entrapment efficiency; however, the difference was not significant, $P>0.05$. Cremophore is a cosurfactant that may increase the solubility of the drug in the surrounding media and facilitate their leakage outside the vesicles. All formulations were stored in the fridge at $5^{\circ} \mathrm{C} \pm 2{ }^{\circ} \mathrm{C}$ for 14 days to address any changes in the amount of confined drugs over the period of storage. Although the liposomes were kept in PBS, which is considered a weak solvent for cinnarizine; surprisingly, the $\mathrm{EE} \%$ was significantly reduced with storage. The leakage may have happened due to many factors. On top of these factors is the addition of cremophor as a cosurfactant and the stability issues that phospholipid may face during the storage [29]. In this study, one liposomal formulation was prepared without using cremophor at any concentration (L3). Thus, if the leakage was caused due to the increase in the solubility of cinnarizine in the surrounding, the encapsulation efficiency of L3 should not have been affected over the time of storage. However, the

Table 1: Optimisation of the liposomal and proliposomal formulations encapsulating cinnarizine (model hydrophobic small drug) and their physicochemical properties.

\begin{tabular}{|c|c|c|c|c|c|c|}
\hline ID & Cinnarizine, $\mathrm{mg}$ & $\begin{array}{l}\text { Lipid: Cholesterol: } \\
\text { Cremophor ratio }\end{array}$ & Size $(n m)^{a, b}$ & $\mathrm{PDI}^{\mathrm{a}}$ & $\% \mathrm{EE}^{\mathrm{c}, \mathrm{b}}$ & $\%$ Drug release $\mathrm{e}^{\mathrm{d}, \mathrm{b}}$ \\
\hline L1 & 5 & $6: 3: 1$ & $407.50 \pm 13.82$ & 0.137 & $61.68 \pm 3.99$ & $74.30 \pm 1.82$ \\
\hline $\mathrm{L} 2$ & 5 & $5: 4: 1$ & $299.02 \pm 13.55$ & 0.279 & $63.61 \pm 1.25$ & $71.20 \pm 3.97$ \\
\hline L3 & 5 & $5: 5: 0$ & $402.18 \pm 24.04$ & 0.118 & $65.89 \pm 1.44$ & $77.10 \pm 2.94$ \\
\hline PL1 & 5 & $6: 3: 1$ & $668.29 \pm 31.77$ & 0.219 & $60.21 \pm 6.45$ & $101.20 \pm 3.87$ \\
\hline PL2 & 5 & $5: 4: 1$ & $815.26 \pm 19.6$ & 0.288 & $59.48 \pm 1.55$ & $98.36 \pm 3.25$ \\
\hline PL3 & 5 & $5: 5: 0$ & $857.96 \pm 8.99$ & 0.182 & $63.03 \pm 1.24$ & $96.31 \pm 2.91$ \\
\hline
\end{tabular}

${ }^{\mathrm{a}}$ Size and PDI were measured by dynamic light scattering in deionised water. ${ }^{\mathrm{b}}$ Results are expressed as mean \pm SD $(n=3) .{ }^{\mathrm{c}} \%$ Encapsulation efficiency (\%EE) of cinnarizine was determined by measuring absorbance at $\lambda=253 \mathrm{~nm}$ by single-beam UV/V is spectrophotometer (Camspec $\mathrm{M} 501$, UK) against $\mathrm{HCl}$ as blank. \%EE was determined using the following equation: \%EE= (amount of encapsulated drug/initially used drug) * $100 \% .{ }^{\mathrm{d}} \%$ of total cinnarizine released in SGF after $4 \mathrm{~h} \%$ of EE after 14 days of storage in the fridge at $5 \pm 2{ }^{\circ} \mathrm{C}$. Proliposomes were stored in a desiccator with silica beads. 


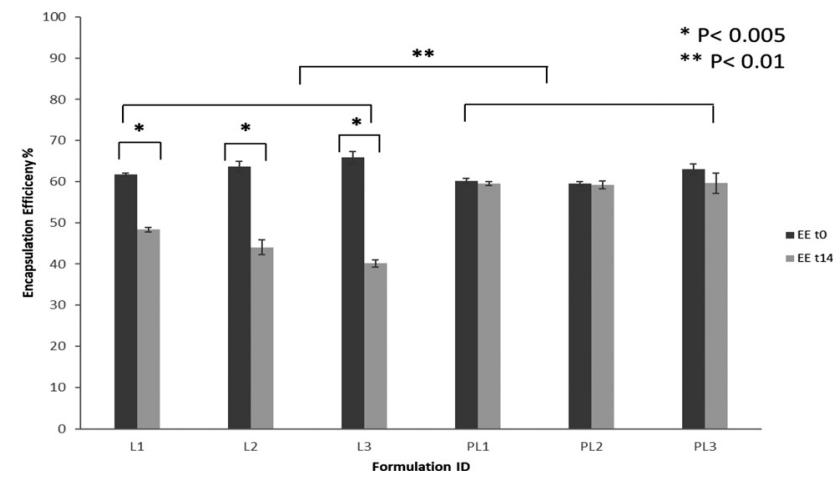

Figure 1: The percentage of the encapsulation efficiency of cinnarizine in the liposomal and proliposomal formulations. The encapsulation efficiency was evaluated on the same day of the preparation and after 14 days of storage in the fridge. The quantification was performed by measuring $A_{253}$ by UV/vis single beam spectrometer. The amount of confined drug was reduced significantly over the period of storage ${ }^{*} P<0.005$. EE $\% t_{14}$ was significantly different between both types of vesicles, ${ }^{\star \star} P<0.01$. No significant difference was reported between $\mathrm{EE} \% t_{0}$ for both liposomes and proliposomes.

percentage of encapsulation efficiency in L3 was depleted significantly during the 14 days of storage, Figure 1 . Also, changing lipid: cholesterol ratio between L1 and L2 did not change the case; it means increasing the amount of cholesterol for the sake of increasing the packing density of liposomes did not protect the liposomes from degradation and, consequently, from leakage. These results confirm that the phospholipid was prone to destabilising events during the time of storage in the fridge. These stabilising events may involve physical and chemical processes or either one.

Proliposomes, the solid powder, kept the same amount of the entrapped drug over the period of storage. These unchanged values are expected as the solid systems usually have zero energy, then no release kinetics may occur; thus, keeping the same amount of drug inside the vesicles. Also, the multilamellar nature of proliposomes provided wide space inside the double layers for confining lipophilic drugs and more distance for leakage; hence, low leakage happened.

Drug release was investigated in SGF, Figure 2. Two different patterns were noticed between the liposomal and proliposomal formulations. Cinnarizine was released from the liposomal vesicles, relatively, very quickly. After $90 \mathrm{~min}$, around $70 \%$ of the drug was released, and the level of the released drug remained around the same level. Reaching a plateau confirms that the drug was diffused from the area between the bilayer in a saturable manner. To clarify, after the dissolution of $70 \%$ of the drug, the surrounding dissolution medium reached saturation without the ability to dissolve any further amount of drug beyond this. Nevertheless, in proliposomes, the pattern was completely different; the drug release reached $100 \%$ after 2-4. The sustained-release manner was recorded; the relationship between the time and the percentage of the released drug was linear. In multilamellar phospholipid vesicles, the hydrophobic drugs are confined into the interlayer spaces; therefore, at the beginning of the release process, the drug that exists inside the outer space area was diffused and followed gradually by the drug from the other inter areas. Also, the large particle size of the proliposomes means a less total surface area the system has; thus, slower drug release may occur. When a drug is dissolved gradually, but not suddenly, it has a higher chance to be dissolved due to the sink condition and the continuous renewal of the dissolution media. Also, many studies unveiled the mechanism of increasing the solubility of the entrapped lipophilic drugs inside proliposomes. Hiremath et al. concluded that proliposomes have the ability to convert the physical nature of the loaded hydrophobic drug from a crystalline into a more amorphous agent, which consequently enhances the dissolution profile [23].

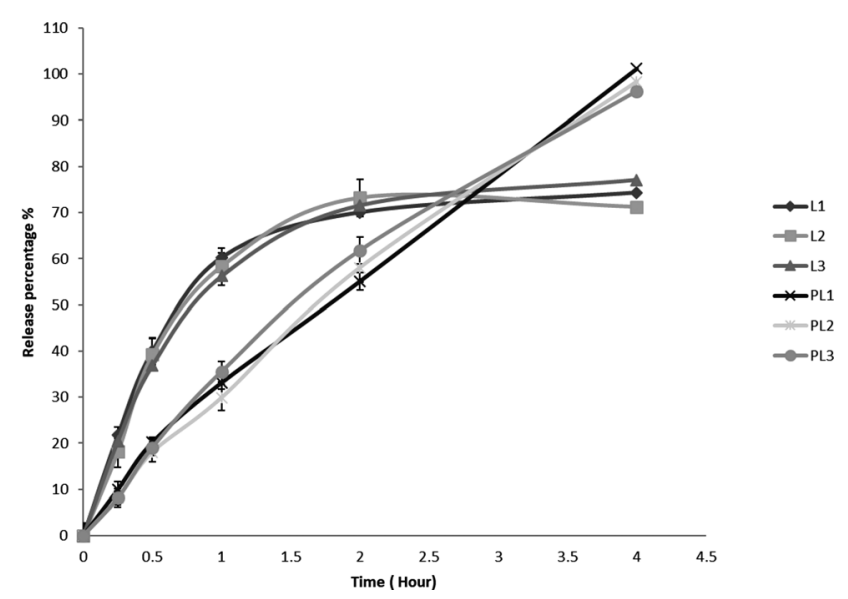

Figure 2: In vitro release profile of cinnarizine from the liposomes and proliposomes in simulated gastrointestinal fluids (without enzyme) at $37^{\circ} \mathrm{C}$. Cinnarizine release from the formulations was determined in simulated gastric fluids (SGF) for 4. The SGF was prepared according to the British Pharmacopeia; however, no digestive enzymes were added. Drug release was determined by quantification of cinnarizine amount in SGF by reading $A_{253}$ after centrifugation of dissolution vesicles and collecting the supernatant. The release profile of the drug from the liposomal formulations was saturable; it reached around $70 \%$ after $2 \mathrm{~h}$ and remained the same for the rest of the experiment. However, the proliposomes released the drug in a sustained pattern throughout the entire time of the experiment; this confirms the entrapment of the drug in the hydrophobic domain between the layers of multilamellar vesicles of proliposomes. 


\section{Conclusion}

This study shows a successful case of enhancing the bioavailability of cinnarizine by utilising proliposomes as an oral drug carrier. Cinnarizine belongs to class II, where drugs have good permeation and poor solubility as a main barrier for bioavailability. Liposomes could benefit the clinical uses of cinnarizine by enhancing its dissolution profile and its pharmacokinetic properties. However, liposomes are facing many stability challenges, which may burden their storage. Therefore, proliposomes are pioneered to be dry stored and reconstituted upon usage. Here, we formulated three different proliposomes and three liposomes for the oral delivery of cinnarizine with the same ingredients. Various physicochemical characteristics, drug release, and storage stability were investigated. Although both approaches utilised the same method of preparation which ended up with the same encapsulation efficiency, significant differences were reported in term of drug release and stability over 14 days of storage. Proliposomes have shown superiority to their liposomal counterparts in term of stability and drug release. Proliposomes showed slower and extended-release in comparison to the liposomal formulations. In proliposomes, the entire encapsulated drug was released over the time of the experiment $(4 \mathrm{~h})$. In conclusion, proliposomes may play a crucial role, as an oral delivery system, in comparison with liposomes, which is owed to their ability to achieve full and sustained drug release in SGF and withstand over the entire period of storage with full protection of loaded drugs. Hence, proliposomal formulations have established their position in oral drug delivery systems, and this study may open new horizons for many researchers to further investigate this field to improve the bioavailability of many poorly soluble drugs. In order to enhance the feasibility and applicability of the developed proliposomes, further drying techniques and conditions should be investigated, e.g. lyophilisation or spray drying. Noteworthy, scanning and investigating a range of cryoprotectant is recommended to obtain the most stable formulations.

Research funding: None declared.

Author contribution: All authors have accepted responsibility for the entire content of this manuscript and approved its submission.

Competing interests: Authors state no financial or nonfinancial conflict of interest.

Informed consent: Informed consent was obtained from all individuals included in this study.

Ethical approval: Not applicable.

\section{References}

1. Turner D, Lurie Y, Finkelstein Y, Schmid T, Gopher A, Kleid D, et al. Pediatric cinnarizine overdose and toxicokinetics. Pediatrics 2006;117:e1067-9.

2. Raghuvanshi S, Pathak K. Recent advances in delivery systems and therapeutics of cinnarizine: a poorly water soluble drug with absorption window in stomach. J Drug Deliv 2014;2014:479246.

3. Fagerberg JH, Tsinman O, Sun N, Tsinman K, Avdeef A, Bergstrom CA. Dissolution rate and apparent solubility of poorly soluble drugs in biorelevant dissolution media. Mol Pharm 2010;7: 1419-30.

4. Li BQ, Yang GQ, Fang SH, Gao JY, Gu FM, Dong X, et al. Effect of route of administration on the pharmacokinetics and toxicokinetics of cinnarizine in dogs. Eur J Pharmaceut Sci 2010; 40:197-201.

5. Yeo LK, Olusanya TOB, Chaw CS, Elkordy AA. Brief effect of a small hydrophobic drug (cinnarizine) on the physicochemical characterisation of niosomes produced by thin-film hydration and microfluidic methods. Pharmaceutics 2018;10:185.

6. Jarvinen T, Jarvinen K, Schwarting N, Stella VJ. beta-cyclodextrin derivatives, SBE4-beta-CD and HP-beta-CD, increase the oral bioavailability of cinnarizine in beagle dogs. J Pharm Sci 1995;84: 295-9.

7. Abu Abed OS. Preparation and Evaluation of Liquid and Nanocapsule Formulations containing Biomolecules. Univeristy of Sunderland; 2016.

8. Abu Abed OS, Chaw CS, Williams L, Elkordy AA. PEGylated polymeric nanocapsules for oral delivery of trypsin targeted to the small intestines. Int J Pharm 2021;592:120094.

9. Alam MI, Paget T, Elkordy AA. Formulation and advantages of furazolidone in liposomal drug delivery systems. Eur J Pharmaceut Sci 2016;84:139-45.

10. Abu Abed OS, Chaw C, Williams L, Elkordy AA. Lysozyme and DNase I loaded poly (D, L lactide-co-caprolactone) nanocapsules as an oral delivery system. Sci Rep 2018;8:13158.

11. Bansal S, Aggarwal G, Chandel P, Harikumar SL. Design and development of cefdinir niosomes for oral delivery. J Pharm Bioallied Sci 2013;5:318-25.

12. Khandavilli S, Panchagnula R. Nanoemulsions as versatile formulations for paclitaxel delivery: peroral and dermal delivery studies in rats. J Invest Dermatol 2007;127:154-62.

13. Akbarzadeh A, Rezaei-Sadabady R, Davaran S, Joo SW, Zarghami $\mathrm{N}$, Hanifehpour $\mathrm{Y}$, et al. Liposome: classification, preparation, and applications. Nanoscale Res Lett 2013;8:102.

14. Elsana H, Olusanya TOB, Carr-Wilkinson J, Darby S, Faheem A, Elkordy AA. Evaluation of novel cationic gene based liposomes with cyclodextrin prepared by thin film hydration and microfluidic systems. Sci Rep 2019;9:15120.

15. Deshantri AK, Metselaar JM, Zagkou S, Storm G, Mandhane SN, Fens $M$, et al. Development and characterization of liposomal formulation of bortezomib. Int J Pharm X 2019;1:100011.

16. Yan-yu X, Yun-mei S, Zhi-peng C, Qi-neng P. Preparation of silymarin proliposome: a new way to increase oral bioavailability of silymarin in beagle dogs. Int J Pharm 2006;319:162-8.

17. Najlah M, Said Suliman A, Tolaymat I, Kurusamy S, Kannappan V, Elhissi AMA, et al. Development of injectable PEGylated liposome encapsulating disulfiram for colorectal cancer treatment. Pharmaceutics 2019;11:610. 
18. Elhissi A. Liposomes for pulmonary drug delivery: the role of formulation and inhalation device design. Curr Pharmaceut Des 2017;23:362-72.

19. Gangadhar V, Gangadharappa HV, Nagashubha B, Balamuralidhara V. Review on novel carrier system: liposomes and proliposomes. Drug Deliv Lett 2014;4:96-109.

20. Byeon JC, Lee SE, Kim TH, Ahn JB, Kim DH, Choi JS, et al. Design of novel proliposome formulation for antioxidant peptide, glutathione with enhanced oral bioavailability and stability. Drug Deliv 2019;26:216-25.

21. Elhissi A, O'Neill MAA, Ahmed W, Taylor KMG. High-sensitivity differential scanning calorimetry for measurement of steroid entrapment in nebulised liposomes generated from proliposomes. [Internet]. Micro \& Nano Lett 2011;6:694-7. Available from: https://digital-library.theiet.org/content/ journals/10.1049/mnl.2011.0086.

22. Porter CJ, Trevaskis NL, Charman WN. Lipids and lipid-based formulations: optimizing the oral delivery of lipophilic drugs. Nat Rev Drug Discov 2007;6:231-48.

23. Hiremath PS, Soppimath KS, Betageri GV. Proliposomes of exemestane for improved oral delivery: formulation and in vitro evaluation using PAMPA, Caco-2 and rat intestine. Int J Pharm 2009;380:96-104.
24. Chu C, Tong SS, Xu Y, Wang L, Fu M, Ge YR, et al. Proliposomes for oral delivery of dehydrosilymarin: preparation and evaluation in vitro and in vivo. Acta Pharmacol Sin 2011;32: 973-80.

25. Najlah M, Jain M, Wan KW, Ahmed W, Albed Alhnan M, Phoenix $\mathrm{DA}$, et al. Ethanol-based proliposome delivery systems of paclitaxel for in vitro application against brain cancer cells. J Liposome Res 2018;28:74-85.

26. Magarkar A, Dhawan V, Kallinteri P, Viitala T, Elmowafy M, Rog T, et al. Cholesterol level affects surface charge of lipid membranes in saline solution. Sci Rep 2014;4:5005.

27. Gu CH, Rao D, Gandhi RB, Hilden J, Raghavan K. Using a novel multicompartment dissolution system to predict the effect of gastric $\mathrm{pH}$ on the oral absorption of weak bases with poor intrinsic solubility. J Pharm Sci 2005;94: 199-208.

28. Danaei M, Dehghankhold M, Ataei S, Hasanzadeh Davarani F, Javanmard R, Dokhani A, et al. Impact of particle size and polydispersity index on the clinical applications of lipidic nanocarrier systems. Pharmaceutics 2018;10:57.

29. Lee MK. Liposomes for enhanced bioavailability of waterinsoluble drugs: in vivo evidence and recent approaches. Pharmaceutics 2020;12:264. 